\title{
Mycobacterium abscessus subsp. massiliense infection secondary to traumatic anterior cruciate ligament reconstruction surgery
}

Christopher P. Emerson 1, Hogan Brecount 1, Daniel Quintero 1, Dylan N. Greif 1, Jean Jose 2, Lee D. Kaplan 1

\begin{abstract}
${ }^{1}$ University of Miami Sports Medicine Institute, University of Miami Miller School of Medicine, Coral Gables, FL, USA.

2 Department of Radiology, University of Miami Miller School of Medicine, Jackson Memorial Hospital, Miami, FL, USA.
\end{abstract}

*Corresponding author: Daniel Quintero. 5000 University Dr Suite 3100, Coral Gables 33146 FL, USA. Phone: +1 (786)-518-9807.E-mail: dxq62@med.miami.edu.

Research Ethics Committee Approval (if necessary): We declare that the patient approved the case report publishing by signing an informed consent form and the case report followed the ethical guidelines established by the Declaration of Helsinki.

Received on: Sep 6, 2021. Accepted on: Sep 29, 2021. Available online: Oct 7, 2021.

\begin{abstract}
Non-tuberculous Mycobacterium (NTM) infections following knee surgery are rarely reported in the literature. One month after arthroscopic lysis of adhesions from anterior cruciate ligament (ACL) reconstruction, a 67-year-old male patient presented with symptoms suggesting septic arthritis. Empiric antibiotics were initiated. Intraoperative cultures were positive for Mycobacterium abscessus. Organismspecific antimicrobial therapy with azithromycin, amikacin, and imipenem were started. Four months later the patient was switched to oral outpatient treatment with azithromycin, clofazimine and bedaquiline. Mycobacterium infections following ACL reconstruction are exceedingly rare in the United States. Successful management of Mycobacterium abscessus infection is exceptionally challenging for both orthopedists and infection disease specialists.
\end{abstract}

Keywords: Anterior Cruciate Ligament Reconstruction; Mycobacterium Abscessus; Osteomyelitis; Septic Arthritis; Graft Removal.

\section{Introduction}

Postoperative anterior cruciate ligament (ACL) reconstruction infections are rare, with postoperative infection rates ranging from $0.14-2.6 \%$ [1]. The causative microorganisms are typically part of the normal skin flora, 
Coagulase-negative Staphylococci and Staphylococcus aureus comprising nearly $90 \%$ of the cases [2]. Graft choice may be associated to the risk of infection with a reported increased risk for hamstring tendon (HT) compared to bone-patellar tendon-bone (BPTB) autograft [3] and for allografts compared to autografts [4].

Mycobacterium infections following ACL reconstruction are rare, with rates of Mycobacterium tuberculosis infection following ACL reconstruction estimated to be $0.68 \%$ in immunocompetent individuals [5]. Nontuberculous Mycobacterium (NTM) infections following knee surgery are also rarely reported in the literature [6, 7]. Herein, we present a case of Mycobacterium abscesses following ACL reconstruction surgery.

\section{Case report}

A 67-year male with no previous medical history, no relevant surgical history and no co-morbidities sustained a traumatic right knee injury after skiing, with preoperative examination performed 10 days after the injury revealing anterior knee instability with knee range of motion of $5-120^{\circ}$. Magnetic resonance imaging (MRI) confirmed the presence of a complete ACL tear and associated medial meniscus horizontal tear within the mid-body.
The patient underwent ACL reconstruction with an all-soft tissue quadriceps tendon (QT) autograft and partial medial meniscectomy one month after initial injury. Suspensory fixation was used for the femoral side and a poly-ether-ether-ketone interference screw was used for the tibial side. Standard ACL rehabilitation protocol was ordered.

Postoperatively, despite physical therapy (PT) and use of continuous passive motion (CPM) device, the patient's range of motion (ROM) did not improve eight weeks following surgery. Furthermore, the patient had a knee extension deficit of $10^{\circ}$ while knee flexion was limited to $80^{\circ}$. Of note, there were no signs of infection at this time. Two months after surgical intervention, the patient underwent arthroscopic lysis of adhesion (LOA) and manipulation under anesthesia (MUA).

Intraoperatively, there were no signs of infection nor extensive intraarticular adhesions, and passive ROM was $0-125^{\circ}$. Despite the above procedures with PT and use of a CPM machine, the patient's ROM restriction persisted. During the second postoperative week, the patient developed fever, and right knee swelling, warmth and erythema. An arthrocentesis was performed for fluid analysis; however, a dry tap was obtained. The patient was started on 
empiric ciprofloxacin and vancomycin for 14 days.

Lack of clinical improvement and persistent low-grade fever prompted a new MRI evaluation that showed findings suggestive of septic arthritis (Figure 1).

The patient was then admitted to the hospital, where vital signs showed a fever of $38.06^{\circ}$ C. There was no leukocytosis, however C-reactive protein (CRP) and erythrocyte sedimentation rate were elevated at 3.6 $\mathrm{mg} / \mathrm{L}$ and $105 \mathrm{~mm} / \mathrm{h}$ respectively. 2 months after arthroscopic lysis of adhesions, the patient underwent surgical irrigation, arthroscopic debridement of the graft, synovectomy, open debridement of peri-articular soft tissues, and open tibial tunnel debridement with removal of deep implants (Figure 2). Intraoperatively, $15 \mathrm{~mL}$ of purulent joint fluid was obtained and sent for cultures (Figure 2).

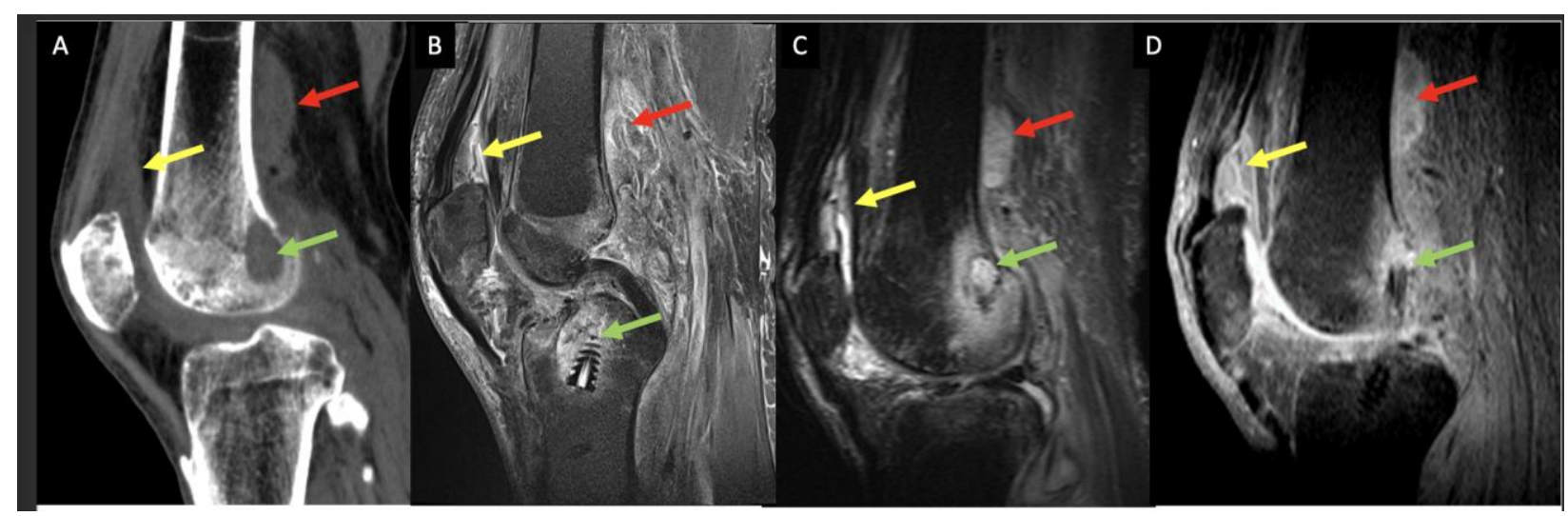

Figure 1. (A) Sagittal computed tomography, (B) and (C) Sagittal Proton Density MRI, and (D) Sagittal T1 Fat Suppressed MRI with IV contrast demonstrate post ACL reconstruction changes, with complex joint effusion and synovitis (yellow arrows), periarticular complex heterogeneous fluid collection along the posterolateral aspect of the distal femur (red arrows), as well as osteolysis of the femoral and tibial ACL surgical tunnels with surrounding bone marrow edema pattern and marrow enhancement (green arrows). Findings are consistent with septic arthritis, periarticular abscess and osteomyelitis.

Intraoperative cultures were positive for Mycobacterium abscessus subsp. Massiliense, as we routinely test for NTM species in knee sepsis cases. The isolate was sensitive to amikacin and clarithromycin, tentatively sensitive to azithromycin and tigecycline and clofazimine, and intermediate to cefoxitin and imipenem. Organismspecific antimicrobial therapy with azithromycin (500mg PO daily), Amikacin (10mg/kg IV daily) and imipenem (500mg every 8h.) was started. Clinical improvement with resolution of fever, warmth, and erythema soon followed. 
One month after surgery, MRI demonstrated a partially retained tibial interference screw, decreased effusion, synovial thickening, enhancement, and adjacent periosteal reaction, especially posterior to the distal femur and in the suprapatellar recess (Figure 3).
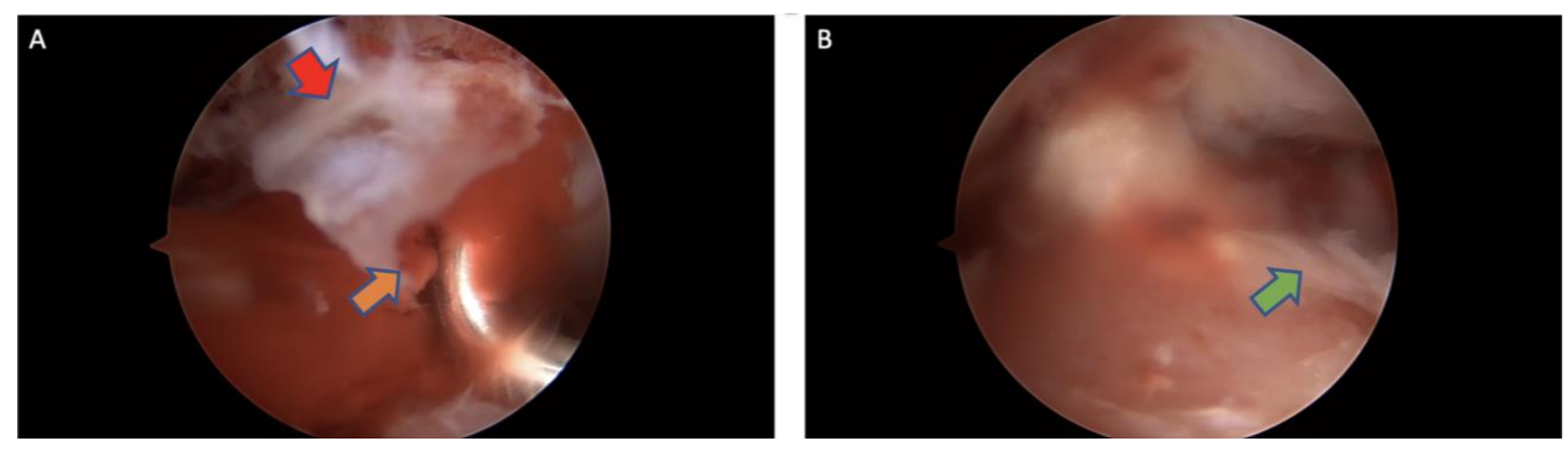

Figure 2. A/B. Arthroscopic image demonstrating extensive hyperemia (confluent redness image A) and synovitis around the ACL graft (red arrow). These findings warranted debridement of the graft (orange arrow demonstrates mechanical removal and green arrow demonstrates residual tissue during debridement), synovectomy, open debridement of surrounding peri-articular soft tissues and the tibial tunnel with removal of deep implants.

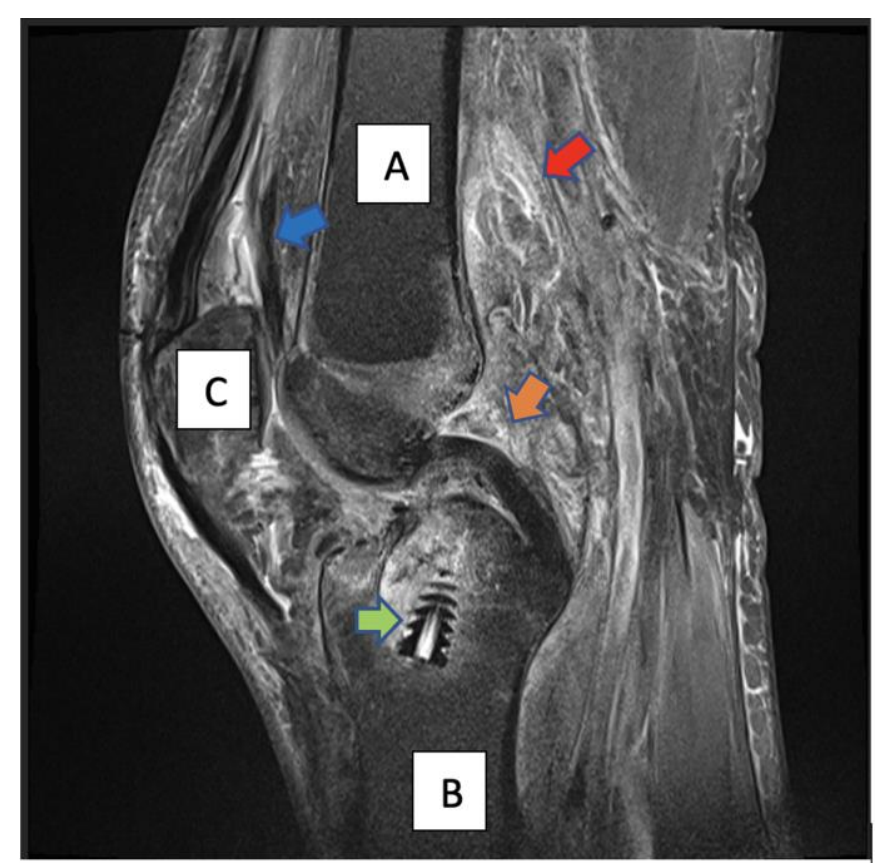

Figure 3: Sagittal proton density fat suppressed MRI demonstrating surgical debridement of the ACL graft (orange arrow), with retention of the tibial interference screw (green arrow). Compared to prior MRI (figure 1) the effusion, synovial thickening, and periosteal reaction are reduced in both the suprapatellar pouch (blue arrow) and along the posterior femur (red arrow). For orientation: A. symbolizes the femur, C. patella and B. Tibia. 
The bone marrow edema and corresponding confluent low T1 signal within the anterior tibia and adjacent to the tibial tunnel appeared to be worse than the MRI done one month prior and was concerning for ongoing osteomyelitis. On month after surgical irrigation, the patient underwent removal of the partially retained tibial screw, irrigation, and tunnel reaming/debridement (Figure 4). Specimens sent for Mycobacterium PCR and acid-fast bacilli cultures were negative.

In addition, he was continued on azithromycin (500mg daily), the amikacin was switched to tigecycline (50 mg IV every 12h) after he developed ototoxicity from the amikacin and the imipenem was narrowed to cefoxitin (3g
IV every 8hrs.). Three months after removal of tibial screw and tunnel reaming the cefoxitin was discontinued after he developed a generalized rash associated with eosinophilia (18.7\%) and he was kept on azithromycin and tigecycline.

As of recent, the patient displayed range of motion of 5-110 degrees at their follow up visit two months after cefoxitin was discontinued he was switched to a complete oral antibiotic that included azithromycin, clofazimine and bed aquiline. This regimen was continued for 7 months after which the patient returned to our clinic having ceased all anti-biotic treatment and continued to progress through our standard rehabilitation protocol.

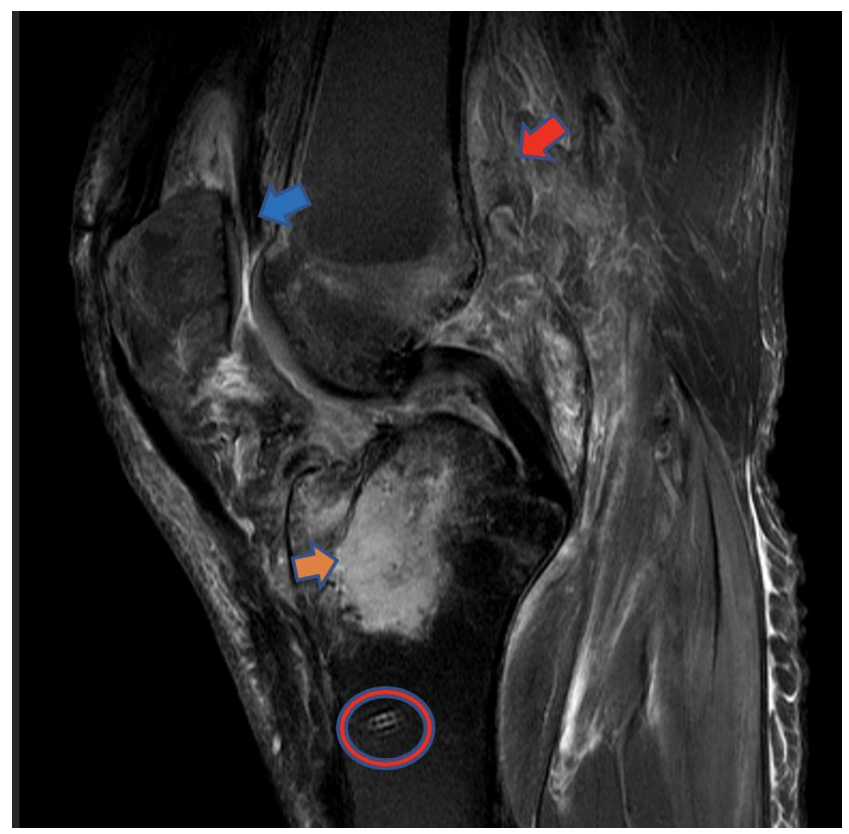

Figure 4: Sagittal proton density fat suppressed MRI demonstrates interval removal of the previously seen tibial tunnel interference screw (orange arrow) since the prior study (figure 3). There remains the ACL Swivelock anchor (red circle), which may serve as a 
nitus for infection. Peri-articular soft tissue edema is still present but improving compared to previous imaging (red arrow and blue arrow).

\section{Discussion and Conclusion}

Mycobacterium infection following ACL reconstruction is rare. Nag et al. [5] reported a series of eight $M$. Tuberculosis infections following autograft ACL reconstruction. Seven patients had reconstruction with the HT graft, while one patient had infection with the BPTB graft [5].

NTM infection following ACL reconstruction is also extremely rare [6, 7]. NTM are common environmental organisms that can survive harsh conditions (soil and aquatic environments), including low $\mathrm{pH}$ and extreme temperatures, and can form biofilms. NTM species are reportedly more likely to occur in immunosuppressed patients and at the site of a prior musculoskeletal procedure [8].

M. abscessus is one of the most common cause of NTM infection and in the United States and its prevalence is increasing [9]. Recent findings support its taxonomic status as a single species comprising three subspecies designated abscessus, bolletii and massiliense [10]. $M$. abscessus, which when cultured is phenotypically similar to other NTM species, has proven to be difficult to identify in the laboratory (traditional culture methods for $M$. Tuberculosis are routinely used, which are too harsh nullify the yield of NTM) and eradicate due to limited antimicrobial susceptibility, lack of bactericidal options, its ability to form biofilms and the variable presence of the erm (41) gene, which yields inducible resistance to macrolides [11].

M. abscessus possesses a unique species-specific repertoire of genes that confers extreme antibiotic resistance, resulting in cure rates below $50 \%$ even with powerful long duration combination antibiotic treatments such as amikacin and clarithromycin [12].

Furthermore, there are no established treatment guidelines for mycobacterial joint infections. For NTM osteomyelitis, the antibiotic treatment should be continued for a minimum of six months and is sometimes given for one year or longer [9]. There are few reports of $M$. abscessus infection following orthopaedic surgery [13] and with some cases of successful treatment following M. abscessus infection in total hip and knee arthroplasty [7, 14].

Additionally, there has only been one previous reported case of reported of $\mathrm{M}$. abscessus infection after ACL reconstruction [15]. Given the lack of improvement in both that case and our 
case, the graft and implants were eventually removed in an effort to cure the infection. Initially, we tried empiric antibiotic therapy after clinical suspicion of septic arthritis to avoid removal of the unincorporated ACL graft. This case re-demonstrates that septic arthritis is a clinical diagnosis and that surgical irrigation and debridement is warranted upon clinical suspicion. In addition, given the powerful antibiotic resistance of $M$. abscessus, the focus of treatment should be on removal of all implants for early source control. Though no consensus exists on how to manage these patients, we recommend a prolonged course of antibiotics and removal of hardware, with a revision planned after the course of antibiotics has concluded and no clinical signs of infection are present. When the revision is performed, the surgeon should first look for hyperemia and synovitis before continuing with the surgery, and intraoperative cultures and biopsies should be obtained to confirm resolution of infection.

M. abscessus is not susceptible to antibiotics such as ciprofloxacin and vancomycin that are routinely given empirically for bone and joint infections. A non-diagnostic arthrocentesis was the rationale for starting these antibiotics. Two weeks later the patient was readmitted for worsening symptoms and intraoperative cultures drawn were diagnostic, showing positive for $M$. abscessus infection, and allowing the initiation of proper antibiotic treatment against M. abscessus. This highlights the importance of bacteria identification (by routinely ordering both tuberculoid and non-tuberculoid mycobacterial cultures) prior to starting antibiotics in order to improve patient outcomes.

In conclusion, albeit rare, atypical Mycobacterium infections, including Mycobacterium abscessus infection, should be considered in the differential diagnosis when managing infections secondary to anterior cruciate ligament reconstruction surgery. In contrast to most cases, where graft removal is only recommended if infection is persistent after initial debridement and antibiotic therapy, in cases of M. abscessus, we strongly recommend early removal of all implants once the organism is identified. Therefore, via early detection, organism-specific antimicrobial therapy, and early removal of all implants, we can maximize positive outcomes for $M$. abscessus infections secondary to arthroscopic ACL reconstruction.

\section{References}

[1] Gobbi A, Karnatzikos G, Chaurasia S, Abhishek M, Bulgherhoni E, Lane J. Postoperative Infection After Anterior Cruciate Ligament Reconstruction. Sports Health. 2016 Mar-Apr;8(2):187-9. doi: $10.1177 / 1941738115618638$. 
[2] Pérez-Prieto D, Trampuz A, TorresClaramunt R, Eugenia Portillo M, PuigVerdié L, Monllau JC. Infections after Anterior Cruciate Ligament Reconstruction: Which Antibiotic after Arthroscopic Debridement? J Knee Surg. 2017 May;30(4):309-313. doi: 10.1055/s0036-1584559.

[3] Murphy MV, Du DT, Hua W, Cortez KJ, Butler MG, Davis RL, DeCoster TA, Johnson L, Li L, Nakasato C, Nordin JD, Ramesh M, Schum M, Von Worley A, Zinderman C, Platt R, Klompas M. Risk Factors for Surgical Site Infections Following Anterior Cruciate Ligament Reconstruction. Infect Control Hosp Epidemiol. 2016 Jul;37(7):827-33. doi: 10.1017/ice.2016.65.

[4] Palazzolo A, Rosso F, Bonasia DE, Saccia F, Rossi R; Knee Committee SIGASCOT. Uncommon Complications after Anterior Cruciate Ligament Reconstruction. Joints. 2018 Nov 30;6(3):188-203. doi: 10.1055/s-00381675799.

[5] Nag HL, Neogi DS, Nataraj AR, Kumar VA, Yadav CS, Singh U. Tubercular infection after arthroscopic anterior cruciate ligament reconstruction. Arthroscopy. 2009 Feb;25(2):131-6. doi: 10.1016/j.arthro.2008.09.009.

[6] Oh HL, Chen DB, Seeto BG, Macdessi SJ. Mycobacterium fortuitum infection after anterior cruciate ligament reconstruction using a polylactic acid bioabsorbable screw: Case report. Knee. 2010 Mar;17(2):176-8. doi: 10.1016/j.knee.2009.08.004.

[7] Spanyer JM, Foster S, ThumDiCesare JA, Kwon YM, Burke DW, Nelson SB. Mycobacterium abscessus: A Rare Cause of Periprosthetic Knee Joint Infection. Am J Orthop (Belle Mead NJ). 2018 Sep;47(9). doi: 10.12788/ajo.2018.0077.

[8] Goldstein N, St Clair JB, Kasperbauer $\mathrm{SH}$, Daley $\mathrm{CL}$, Lindeque $\mathrm{B}$. Nontuberculous Mycobacterial Musculoskeletal Infection Cases from a Tertiary Referral Center, Colorado, USA. Emerg Infect Dis. 2019 Jun;25(6):1075-1083. doi: 10.3201/eid2406.181041.

[9] Griffith DE, Aksamit T, Brown-Elliott BA, Catanzaro A, Daley C, Gordin F, Holland SM, Horsburgh R, Huitt G, Iademarco MF, Iseman $\mathrm{M}$, Olivier $\mathrm{K}$, Ruoss S, von Reyn CF, Wallace RJ Jr, Winthrop K; ATS Mycobacterial Diseases Subcommittee; American Thoracic Society; Infectious Disease Society of America. An official ATS/IDSA statement: diagnosis, treatment, and prevention of nontuberculous mycobacterial diseases. Am J Respir Crit Care Med. 2007 Feb 
15;175(4):367-416.

doi:

10.1164/rccm.200604-571ST.

[10] Mougari F, Guglielmetti L, Raskine L, Sermet-Gaudelus I, Veziris N, Cambau E. Infections caused by Mycobacterium abscessus: epidemiology, diagnostic tools and treatment. Expert Rev Anti Infect Ther. 2016 Dec;14(12):1139-1154. doi: 10.1080/14787210.2016.1238304.

[11] Brown-Elliott BA, Molina S, Fly T, Njie O, Stribley $P$, Stephenson D, Wallace RJ Jr, Perry JD. Evaluation of a novel rapidly-growing mycobacteria medium for isolation of Mycobacterium abscessus complex from respiratory specimens from patients with bronchiectasis. Heliyon. 2019 Oct 24;5(10):e02684. doi: 10.1016/j.heliyon.2019.e02684.

[12] Hurst-Hess K, Rudra P, Ghosh P. Mycobacterium abscessus WhiB7 Regulates a Species-Specific Repertoire of Genes To Confer Extreme Antibiotic Resistance. Antimicrob Agents Chemother. 2017 Oct 24;61(11):e0134717. doi: 10.1128/AAC.01347-17.

[13] Kim M, Ha CW, Jang JW, Park YB. Rapidly growing non-tuberculous mycobacteria infection of prosthetic knee joints: A report of two cases. Knee. 2017 Aug;24(4):869-875. doi: 10.1016/j.knee.2017.04.015.
[14] Petrosoniak A, Kim P, Desjardins M, Lee BC. Successful treatment of a prosthetic joint infection due to Mycobacterium abscessus. Can J Infect Dis Med Microbiol. 2009 Fall;20(3):e94-6. doi: 10.1155/2009/968052.

[15] Ng SW, Yee Han DL. Lessons learnt from an atypical mycobacterium infection post-anterior cruciate ligament reconstruction. Clin Orthop Surg. 2015 Mar;7(1):135-9. doi: 10.4055/cios.2015.7.1.135.

Conflict of interest: The author declares no conflicts of interest.

\section{Acknowledgements: None.}

Funding: None.

How to cite this article: Emerson $C P$, Brecount H, Quintero D, Greif DN, Jose J, Lee D. Kaplan LD. Mycobacterium abscessus subsp. massiliense infection secondary to traumatic anterior cruciate ligament reconstruction surgery. Brazilian Journal of Case Reports. 2021 Oct-Dec;01(4):22-30. 